\title{
Choroidal Thickness and Granulocyte Colony- Stimulating Factor in Tears Improve the Prediction Model for Coronary Artery Disease
}

Jose Lorenzo Romero-Trevejo

University of Malaga

Lourdes Fernandez-Romero

Instituto de Investigación Biomédica de Málaga

Josue Delgado

Instituto de Investigación Biomédica de Málaga

\section{Erika Muñoz-Garcia}

Hospital Clínico Universitario Virgen de la Victoria

\section{Andres Sanchez-Perez}

Hospital Clínico Universitario Virgen de la Victoria

Mario Gutierrez-Bedmar ( $\boldsymbol{D}$ bedmar@uma.es )

University of Malaga

Manuel Francisco Jimenez-Navarro

University of Malaga

\section{Research Article}

Keywords: Coronary artery disease, choroidal thickness, granulocyte colony-stimulating factor, cardiovascular prevention, ROC curves, predictive model

Posted Date: January 11th, 2022

DOI: https://doi.org/10.21203/rs.3.rs-1232993/v1

License: (c) (1) This work is licensed under a Creative Commons Attribution 4.0 International License. Read Full License 


\section{Abstract}

Background: Coronary artery disease (CAD) detection in asymptomatic patients still remains controversial. The aim of our study was to evaluate the usefulness of ophthalmologic findings as predictors of the presence of CAD when added to cardiovascular classic risk factors (CRF) in patients with acute coronary cardiopathy suspicion.

Methods: After clinical stabilization, 96 patients with acute coronary cardiopathy suspicion were selected and divided in two groups: 69 patients with coronary lesions and 27 patients without coronary lesions. Their 192 eyes were subjected to a complete routine ophthalmologic examination. Samples of tear fluid were also collected to be used in the detection of cytokines and inflammatory mediators. Logistic regression models, receiver operating characteristic curves and their area under the curve (AUC) were analysed.

Results: Suggestive predictors were choroidal thickness (CT) (OR: 1.02, 95\% Cl: 1.01-1.03) and tear granulocyte colony-stimulating factor (G-CSF) (OR: 0.97, 95\% Cl: 0.95-0.99). We obtained an AUC of 0.9646 (95\% Cl: 0.928-0.999) when CT and tear G-CSF were added as independent variables to the logistic regression model with cardiovascular CRF: sex, age, diabetes, high blood pressure, hypercholesterolemia, smoking habit and obesity. This AUC was significantly higher $(p=0.003)$ than the prediction derived from the same logistic regression model without CT and tear G-CSF (AUC $=0.828,95 \%$ Cl: 0.729-0.927).

Conclusions: CT and tear G-CSF improved the predictive model for CAD when added to cardiovascular CRF in our sample of symptomatic patients. Subsequent studies are needed for validation of these findings in asymptomatic patients.

\section{Background}

To date, there have been no in-depth studies into the relationship between ophthalmological findings and coronary artery disease (CAD), which may seem paradoxical considering the anatomical and physiopathological characteristics shared by both systems ${ }^{1,2}$. Cardiovascular diseases (CVD) are the leading cause of death worldwide, and accounted for almost 18 million deaths according to estimations made in $2017^{3}$. During that year, the estimate established that within the 54 countries of Europe and the Mediterranean represented in the European Society of Cardiology, almost 109 million people lived with some type of CVD. The most common of these diseases, which affected 35 million people, was CAD. On the other hand, almost 20 million new cases of CVD were also diagnosed, of which 3.6 million were classified as CAD ${ }^{4}$. However, the global burden of CVD is not just a health issue; for health systems, it represents a financial challenge that is expected to undergo exponential growth in the future ${ }^{5}$. For these reasons, the availability of predictive tools that would help reduce the prevalence and incidence rates of CVD are a key element to improve both patient health and the sustainability of healthcare services, 
bearing in mind the large number of people who would benefit no matter how small the preventive measure to apply.

Since the Framingham Heart Study identified the classic risk factors (CRF) for developing CVD ${ }^{6}$, numerous cardiovascular risk prediction models have received validation and gained popularity. These models consider factors such as the presence of high blood pressure, hypercholesterolemia, diabetes, obesity, smoking habit, alcohol consumption, diet and physical exercise among others ${ }^{7}$. This group of factors constitutes more than $90 \%$ of attributable risk for onset of CAD among the population ${ }^{8}$, which is why controlling or eliminating them would imply a substantial reduction in the incidence of this disease. Nevertheless, despite acceptable achievements in many CRF, in our context the predominance of obesity and especially diabetes has increased two to three times during the last 30 years, making them the two most important factors to control if we are to reach the objectives proposed by the World Health Organization for the year $2025^{9}$. The prediction models available to date have manifest limitations, chiefly because their correct application among all populations worldwide is not possible, and it would be ideal if each community could develop its own model ${ }^{10}$, or because they have difficulty detecting the risk, for instance, among women. For these reasons, screening to detect asymptomatic CAD still remains controversial, and updated guidelines continue to emphasise the need for its early detection in order to establish prognostic and therapeutic strategies designed to reduce morbidity and mortality among such patients ${ }^{11}$.

Its particular structure and the ease of access to the eye make this organ a candidate for obtaining potentially useful parameters in this sense, bearing in mind its involvement in a multitude of systemic processes whose first sign may even show itself as an ophthalmological symptom ${ }^{12}$. Recently, ophthalmologic findings have been associated with preclinical neurologic conditions as Alzheimer's disease ${ }^{13,14}$. Moreover, changes in the cardiovascular system have also been related with signs that are visible in the eye, making this organ a window that provides quick access to the cardiovascular system thanks to the ease with which it is possible to see findings ${ }^{15}$. Finally, considering that some CRF for CVD such as diabetes, high blood pressure or hyperlipidaemia, produce typical, specific lesions in the eye fundus, there is a clearly established relationship between this organ and cardiovascular conditions. Nevertheless, to date, research has shown no clear relationship between CVD and the presence of abnormalities in other ocular structures or the expression of different inflammatory mediators in tears.

The objective of this work was, therefore, to analyse the utility of the ophthalmological findings and biomarkers in tears as predictors for the detection of CAD. This would improve on existing models, enabling such predictors to become a useful tool in habitual clinical practice since they represent an unexpensive and less invasive way to categorize and efficiently manage these patients, not only from the perspective of patient health but also of healthcare resource management.

\section{Methods}




\section{Study population}

Patients of this observational, case-control, single centre study were assessed in the Cardiology and Ophthalmology Departments of the Virgen de la Victoria University Hospital of Malaga between the months of January-March 2019. Inclusion criteria for the study were suspicion of having a coronary heart disease based on presentation of oppressive chest pain at rest or on with an increase in activity or stress, with or without the presence of dyspnoea at the time of the consultation. Exclusion criteria were the existence of advanced kidney failure, defined as a glomerular filtration rate lower than $30 \mathrm{ml} / \mathrm{min}$, suffering from any disease that reduced life expectancy to less than one year, having presented another cardiovascular event before this study, suffering from diabetic retinopathy or amblyopia in either eye or having undergone retinal photocoagulation treatment in the past.

\section{Cardiovascular examination}

To confirm the existence of CAD, the selected patients underwent coronary angiogram and/or a computerised tomography scan of the coronary arteries. Depending on the angiogram results, we defined $C A D$ as such with findings of a minimum involvement of $70 \%$ in any major epicardial artery (right coronary, anterior descending or marginal circumflex) or more than $50 \%$ in the left main coronary artery. Based on the computerised tomography of coronary arteries, we defined CAD as such where there was involvement of at least $50 \%$ of any coronary artery. After these results, patients we distributed into two study subject groups: patients without coronary lesions and patients with coronary lesions. After their clinical stabilisation, patients underwent a full ophthalmological examination within the first ten days after the onset of the clinical symptoms that were the reason for the cardiovascular study.

\section{Ophthalmological examination}

The same ophthalmologist conducted the ophthalmological examinations at the same time of day (15 $\mathrm{pm}$ to $18 \mathrm{pm}$ ). Before carrying out examinations, we collected data about the CRF for the onset of cardiovascular disease for each patient, such as sex, age, presence of diabetes, high blood pressure, hypercholesterolemia, smoking habit or obesity. The ophthalmological examination consisted of evaluating different clinical parameters, such as best-corrected visual acuity (BCVA) with numerical eye charts, intraocular pressure (IOP) using a Perkins Mk3 tonometer (Haag-Streit, Essex, UK), Schirmer's test (ST) using Schirmer-Plus ${ }^{\circledR}$ strips (GECIS, Neung-sur-Beuvron, France), central corneal thickness (TCCT and $\mathrm{PCCT}$ ), axial length (AL) and several variables obtained by means of optical coherence tomography (OCT) and OCT-angiography. In addition, as laboratory variables, we studied the presence of cytokines and other inflammatory mediators in samples of tears.

Central corneal thickness was automatically measured with the Orbscan® IIz (Bausch \& Lomb, Rochester, USA) topographer and, after administration of tetracaine and oxybuprocaine anaesthetic eye drops, manually with the OcuScan ${ }^{\circledR} \operatorname{RxP}$ (Alcon Laboratories, Texas, USA) ultrasound pachymeter, with calculation of the arithmetic mean of 10 consecutive measurements taken from each eye with the patient seated. AL was automatically obtained with partial coherence interferometry using the IOLMaster®500 
(Carl Zeiss Meditec AG, Jena, Germany) optical biometer. Cirrus ${ }^{\text {TM }}$ (Carl Zeiss Meditec AG, Jena, Germany) high-definition (HD)-OCT was used for OCT, with the equipment providing automatically obtained values for central macular thickness (CMT), macular cube volume (MCV), mean macular thickness (MMT), retinal nerve fibre layer thickness (RNFLT), ganglion cell layer thickness (GCLT) and ganglion cell layer minimum thickness (GCLMT). Choroidal thickness (CT) was calculated manually using the device's ruler from the images it produced, and was defined as the distance between the external hyper-reflective band of the retinal pigment epithelium and the internal hyper-reflective band of the sclera. This was expressed as the arithmetic mean of 10 measurements: 5 on the horizontal axis and 5 on the vertical axis, with a separation of 500 micra between them to either side of subfoveal area. For inclusion in this study, the minimum acceptable image quality was 6 out of 10. The OCT-angiography was performed with DRI OCT Triton $^{\mathrm{TM}}$ plus (Topcon Medical Systems, Oakland, USA) and the device software automatically provided the values for central vascular density in the different layers of the retina (ILM, RNFL-GCL, GCL-IPL, IPL$\mathrm{INL}$ ). In the case of prior corneal surgery or a clinically significant eye cataract, we excluded the values obtained for these eyes for BCVA, IOP, TCCT, PCCT and the OCT and OCT-angiography.

Conducting ST served also to collect tear samples from each patient. The paper strip was placed to rest in the lower base of each eye, without previous application of topical anaesthetic, and left for 5 minutes. The study excluded samples from any eyes where moisture on the strip measured less than $6 \mathrm{~mm}$ after this period. Valid samples were immediately frozen at $-80^{\circ} \mathrm{C}$ after collection, and remained th that temperature until analysis. For protein elution each paper sample was cut into small pieces that were introduced into $100 \mathrm{ml}$ of PBS with Tween ${ }^{\circledR} 20$ at $0.3 \%$, bovine albumin serum at $0.5 \%$ and protease inhibitor, for overnight incubation $4^{\circ} \mathrm{C}$ before collection of supernatant. Measurement of the total amount of protein in each sample used the NanoDrop ${ }^{\mathrm{TM}}$ One (ThermoFischer Scientific, Waltham, MA, USA) spectrophotometer with absorbance measured at $280 \mathrm{~nm}$. Detection of cytokines and inflammatory mediators followed the instructions of the Bio-Plex Pro ${ }^{\mathrm{TM}}$ kit Human Cytokine 27-Plex Assay (Bio-Rad Laboratories, Hercules, CA, USA). The 27 cytokines and inflammatory mediators analysed were interleukin (IL)-1b, IL-1RA, IL-2, IL-4, IL-5, IL-6, IL-7, IL-8, IL-9, IL-10, IL-12p70, IL-13, IL-15, IL-17, basic fibroblast growth factor (FGF), eotaxin (EO), granulocyte colony-stimulating factor (G-CSF), granulocytemacrophage colony-stimulating factor (GM-CSF), interferon (IFN)-g, chemokine (IP)-10, monocyte chemoattractant protein (MCP)-1, macrophage inflammatory protein (MIP)-1a, platelet-derived growth factor (PDGF)-bb, MIP-1b, chemoquine ligand 5 (RANTES), tumour necrosis factor (TNF)-a and vascular endothelial growth factor (VEGF).

\section{Statistical analysis}

Data were analysed with Stata ${ }^{\circledR} 17$ (StataCorp LLC, Texas, USA). Characteristics of participants were described as a mean and standard deviation for continuous variables and percentage for categorical variables. Group comparisons were carried out using a Student's t-test or Chi-squared test as appropriate.

For each variable associated with the presence of coronary lesions in the bivariate analysis, a logistic regression model was estimated that included the CRF: sex, age, diabetes, high blood pressure, 
hypercholesterolemia, smoking habit and obesity. We assessed the statistical significance of each variable using the likelihood-ratio test based on the models with and without the variable (only with CRF). For variables that were statistically significant, we estimated the area under curve (AUC) associated with the logistic regression model. To estimate the predictive capacity of these variables, we compared the areas under curve using the Chi-squared test, taking the AUC of the logistic regression model that only included CRF. Finally, a logistic regression prediction model was constructed that included the CRF and all variables whose logistic regression models showed an AUC higher than the only-CRF model.

\section{Results}

A total of 96 patients were recruited and distributed into the two study subject groups. There were 27 patients without coronary lesions and 69 patients with coronary lesions. The total 192 eyes examined were individually analysed. Table 1 shows the distribution of absolute and relative frequency of the number of eyes included in each group by CRF studied.

Table 2 provides the results of the comparative study between the two groups of patients in terms of the ophthalmological variables assessed. We excluded the cytokines IL-2, IL-10, IL-12p70, IL-15, FGF and VEGF before the study because the laboratory tests found no detectable values of them. After the comparative study, the variables finally chosen to carry out the prediction model because they showed statistically significant differences between the mean values for both groups were axial length, CT, IL-8, GCSF, MCP-1, MIP-1a and RANTES.

The logistic regression study found a direct relationship between CT and RANTES and the presence of coronary lesions, and an inverse relationship between IL-8, G-CSF, MCP-1 and the presence of such lesions. Conversely, the ophthalmological variables that, on an individual basis, significantly improved results of AUC when added to the CRF were CT and G-CSF. Table 3 shows the results of this analysis.

In the multivariate analysis that included CT and G-CSF along with the other CRF, the AUC obtained was 0.964 , which constituted a statistically significant difference in comparison with the AUC value that only considered AUC (Fig. 1, Table 4). The probability of the existence of coronary lesions according to the best model obtained from the studied sample was possible to calculate using the equation In (Odds of lesion) $=-18.56+0.032 \times$ CT $-0.067 \times$ G-CSF $-3.788 x$ sex $+0.1923 x$ age $+2.372 x$ diabetes $+1.696 x$ high blood pressure $-2.425 \mathrm{x}$ hypercholesterolemia $+7.357 \mathrm{x}$ smoking habit $-1.209 \mathrm{x}$ obesity. The receiver operating characteristic (ROC) curve analysis revealed that a $p^{3} .45$ would predict the existence of coronary lesions with a sensitivity of $96.49 \%$ and a specificity of $84 \%$, allowing the correct classification of $92.68 \%$ of patients.

\section{Discussion}

The results of our study demonstrate that, among the patients studied, the values for CT and G-CSF in tears improve the AUC of the prediction model for CAD when added to CRF for this disease. These 
variables have a direct and inverse relationship respectively with the presence of coronary lesions. To the best of our knowledge, this is the first study into the use of two ophthalmological variables, one clinical and the other laboratory-based, to facilitate the detection of CAD. Its added advantage is that obtaining the data is relatively quick, simple and non-invasive in the usual clinical practice.

The choroid consists of a dense network of blood vessels, located between the retina and the sclera, whose function is to supply oxygen and nutrients to the external layers of the retina and pigment epithelium, the subfoveal avascular zone and the optic nerve. In recent years, a growing body of evidence has shown that changes in choroidal microvasculature might be indicative of other systemic diseases that affect blood vessels ${ }^{16,17}$. Thus, the relationship between the choroid and CVD represents an area of clinical interest and a potential prognostic factor or biomarker for such diseases ${ }^{1}$. Although there is growing body of evidence to support the theory that changes in CT may be of prognostic use, controversy still exists in this regard ${ }^{18}$. Recent studies have suggested that in CAD there is a probable relationship between a decrease in capillary density and choroid blood flow, as well as with a general thinning of choroid $^{2,19-21}$. Such reports contradict the findings of our study, which indicate that an increase in choroidal thickness is associated with the presence of coronary lesions. However, there are some important aspects of the above-mentioned studies that may justify the disparity. First, in addition to the fact that the number of patients studied was slightly lower than in our research, the inclusion criteria for two of the studies was the presence of coronary slow-flow phenomenon ${ }^{19}{ }^{20}$, the definition of which excludes the presence of evident obstructive disease and which, therefore, would preclude comparison of the results obtained. Secondly, another of the studies associated the presence of CAD with a decrease of capillary density and choroid flow in general, without referring to thickness ${ }^{21}$. Furthermore, this study shows an unexpectedly more intense vascular density of the external retina among CAD patients, with no determination of whether this increased density could be related with CT or not, an association that still remains uncertain ${ }^{22}$. Finally, the work that bears most comparison with ours has shown a decrease in choroidal thickness among patients with CAD. Although the normal spatial pattern of the choroid was maintained, a significantly lower number of patients were used and the study was performed with patients recruited from primary healthcare and ambulatory clinic outpatients ${ }^{2}$. This means that factors such as the possible effect of a time-lapse between having passed the acute phase of the disease, or daily fluctuations in CT may have had a significant influence on obtaining the data. In any case, it is practically impossible to establish the isolated connection between CAD, CT and clinical risk, as a great number of physiological variations, lifestyle, pharmacological treatment and comorbidities such as high blood pressure, dyslipidaemia, diabetes or other inflammatory and autoimmune conditions have been individually associated with the increase or decrease of clinical risk ${ }^{1,15}$. Given this circumstance, from a clinical perspective, it would be more practical to create and validate prediction models that integrate all of these conditions, as we have undertaken in this work.

G-CSF is a hematopoietic growth factor produced by monocytes, macrophages, fibroblasts, endothelial cells, vascular smooth muscle cells or bone marrow stromal cells that have the capacity to regulate proliferation, differentiation, survival and growth of neutrophil progenitor cells, contributing to the 
conversion of granulocyte colony-forming units to polymorphonuclear leukocytes, with their subsequent release from bone marrow into the bloodstream ${ }^{23}$. In addition, G-CSF mobilises stem cells from bone marrow to peripheral blood, and induces their differentiation to cardiomyocytes or endothelial cells when they come into contact with damaged areas of the myocardium ${ }^{24}$. Thus, in experimental models of acute myocardial infarction, the animals treated with G-CSF showed a significant increase in survival compared to the ones who did not receive it ${ }^{25}$, although other published studies report contradictory findings ${ }^{26}$. Despite the currently existing controversies, having detected that high plasma levels of endogenous GCSF may predict cardiovascular events regardless of the established risk factors ${ }^{27}$, the use of G-CSF as an element that mobilises stem cells to repair myocardial damage, in the treatment of congestive heart failure, or as a mediator in atherosclerosis, is an attractive concept for future research. Such research should particularly focus on the search for feasibility and an acceptable cost-benefit relationship $24,28,29$. To the best of our knowledge, this is the first time that research has associated levels of G-CSF in tears with the presence of CAD, showing that an increase in its levels may constitute a protective factor, whereas lower levels are associated with the existence of a possible onset of coronary lesions. These data concur with the results of these previously published studies that report G-CSF as playing an important role in the cardiac regeneration processes after ischaemic damage, although future tests are necessary to determine with greater clarity which mechanisms are involved in these processes.

The prevention of CVD requires the identification of people who are at high risk, in order to propose suitable pharmacological, dietary or lifestyle interventions. Recent decades have seen the development of a multitude of prediction models for the general population, to name but a few: the Framingham ${ }^{30}$, SCORE $^{31}$, QRISK $^{31}$ or, in our country, IberScore ${ }^{33}$. Nevertheless, in most cases their use remains unclear, due to defects in method, incomplete presentation, lack of external validation or heterogeneity in the chosen predictors, or in the definition of the events evaluated ${ }^{34}$. For this reason, in the age of excessive information, future work should focus on the external validation and comparison of existing models, their combination and adaptation for application among local populations, and their extension by means of adding new predictors ${ }^{34,35}$. To this end, our results propose two new ophthalmological variables: CT and G-CSF in tears. Used in conjunction with the CRF, they offer a prediction model that allows the correct classification of a higher number of patients based on the presence or absence of coronary lesions. Moreover, and thanks to this, transdisciplinary management of these patients would be possible since, in the current context, individuals with CVD do not receive suitable ophthalmological follow-up ${ }^{1}$, and unidisciplinary approach may not be optimal when disease causation is complex and health decisions are pressing ${ }^{36}$.

The main strength of our study resides in the degree of precision in measuring the ophthalmological variables employed. Among its limitations, the foremost are the use of a homogeneous group of patients who come from a single hospital, the relatively small sample size and the fact that it only studied symptomatic individuals. For all of these reasons, future prospective studies with a higher number of patients are necessary to verify these promising results and evaluate the validity of their application in asymptomatic individuals. 


\section{Conclusions}

CT and tear G-CSF improved the predictive model for CAD when added to cardiovascular CRF in our sample of symptomatic patients. Although subsequent studies are needed for validation of these

findings in asymptomatic patients, this is the first study into the use of two ophthalmological variables to facilitate the detection of CAD.

\section{List Of Abbreviations}

AL: Axial length.

AUC: Area under the curve.

BCVA: Best corrected visual acuity.

CAD: Coronary artery disease.

Cl: Confidence interval.

CMT: Central macular thickness.

CRF: Classic risk factors.

CT: Choroidal thickness.

CVD: Cardiovascular diseases.

G-CSF: Granulocyte colony-stimulating factor.

GCL: Ganglion cell layer.

GCLMT: Ganglion cell layer minimum thickness.

GCLT: Ganglion cell layer thickness.

HD: High definition.

IL: Interleukin.

ILM: Inner limiting membrane.

INL: Inner nuclear layer.

IOP: Intraocular pressure.

IP: Chemokine. 
IPL: Inner plexiform layer.

MCP: Monocyte chemoattractant protein.

MCV: Macular cube volume.

MIP: Macrophage inflammatory protein.

MMT: Mean macular thickness.

OCT: Optic coherence tomography.

OR: Odds ratio.

PCCT: Pachymetry central corneal thickness.

PDGF: Platelet-derived growth factor.

RANTES: Chemokine ligand 5.

RNFL: Retinal nerve fibre layer.

RNFLT: Retinal nerve fibre layer thickness.

ROC: Receiver operating characteristic.

SD: Standard deviation.

ST: Schirmer's test.

TCCT: Topography central corneal thickness.

TNF: Tumour necrosis factor.

VEGF: Vascular endothelial growth factor.

\section{Declarations}

\section{Ethics Approval and Consent to Participate}

This study received the approval of Malaga Provincial Research Ethics Committee (1765-N-17), in compliance with the standards established under the 1964 Declaration of Helsinki and with current national legislation. All participants read and signed informed consent before undergoing the various examinations. 
Not applicable.

\section{Availability of data and materials}

Not applicable.

\section{Competing interests}

The authors declare that they have no competing interests.

\section{Funding}

This work was financially supported by Centro de Investigación Biomédica en Red "Enfermedades Cardiovasculares" (CIBERCV) of the Carlos III Health Institute [CB16/11/00360], the Spanish Ministry of Health, and Fondo Europeo de Desarrollo Regional (FEDER).

\section{Authors' contributions}

All authors contributed equally to the study's conception, design, and performance. All authors read and approved the final manuscript.

\section{Acknowledgements}

The authors thank José Manuel García-Campos and Rafael Luque-Aranda, Heads of the Department of Ophthalmology of the Virgen de la Victoria University Hospital, for putting at our disposal all technical devices employed in our work. The authors thank Carolina Lobo, from the Central Research Support Services of the University of Málaga, for her technical support in the tear samples processing and evaluation. This article received support from Malaga University's Chair of Advanced Therapies in Cardiovascular Pathologies (CIF Q-2918001-E).

\section{References}

1. Yeung SC, You Y, Howe KL, Yan P. Choroidal thickness in patients with cardiovascular disease: a review. Surv Ophthalmol. 2020;65:473-86.

2. Ahmad M, Kaszubski PA, Cobbs L, Reynolds H, Smith RT. Choroidal thickness in patients with coronary artery disease. PLoS One. 2017;12:e0175691.

3. GBD 2017 Causes of Death Collaborators. Global, regional, and national age-sex-specific mortality for 282 causes of death in 195 countries and territories, 1980-2017: a systematic analysis for the Global Burden of Disease Study 2017. Lancet. 2018;392:1736-88.

4. GBD 2017 Mortality Collaborators. Global, regional, and national age-sex-specific mortality and life expectancy, 1950-2017: a systematic analysis for the Global Burden of Disease Study 2017. Lancet. 2018;392:1684-735. 
5. Anand SS, Yusuf S. Stemming the global tsunami of cardiovascular disease. Lancet. 2011;377:52932.

6. Mahmood SS, Levy D, Vasan RS, Wang TJ. The Framingham Heart Study and the epidemiology of cardiovascular disease: a historical perspective. Lancet. 2014;383:999-1008.

7. Piepoli MF, Hoes AW, Agewall S, Albus C, Brotons C, Catapano AL, et al. 2016 European Guidelines on cardiovascular disease prevention in clinical practice: The Sixth Joint Task Force of the European Society of Cardiology and Other Societies on Cardiovascular Disease Prevention in Clinical Practice (constituted by representatives of 10 societies and by invited experts) Developed with the special contribution of the European Association for Cardiovascular Prevention \& Rehabilitation (EACPR). Eur Heart J. 2016;37:2315-81.

8. Yusuf S, Hawken S, Ounpuu S, Dans T, Avezum A, Lanas F, et al. Effect of potentially modifiable risk factors associated with myocardial infarction in 52 countries (the INTERHEART study): case-control study. Lancet. 2004;364:937-52.

9. Timmis A, Townsend N, Gale CP, Torbica A, Lettino M, Petersen SE, et al. European Society of Cardiology: Cardiovascular Disease Statistics 2019. Eur Hear J. 2020;41:12-85.

10. Elsner LK, von Jeinsen B, Grün D, Wolter JS, Weferling M, Diouf $K$, et al. Prognostic performance of the ESC SCORE and its German recalibrated versions in primary and secondary prevention. Eur J Prev Cardiol. 2020;27:2166-9.

11. Visseren FLJ, Mach F, Smulders YM, Carballo D, Koskinas KC, Bäck M, et al. 2021 ESC Guidelines on cardiovascular disease prevention in clinical practice: Developed by the Task Force for cardiovascular disease prevention in clinical practice with representatives of the European Society of Cardiology and 12 medical societies with the special contribution of the European Association of Preventive Cardiology (EAPC). Eur Heart J. 2021;42:3227-337.

12. Kemeny-Beke A, Szodoray P. Ocular manifestations of rheumatic diseases. Int Ophthalmol. 2020;40:503-10.

13. O’Bryhim BE, Apte RS, Kung N, Coble D, Van Stavern GP. Association of preclinical Alzheimer disease with optical coherence tomographic angiography findings. JAMA Ophthalmol. 2018;136:1242-8.

14. Byun MS, Park SW, Lee JH, Yi D, Jeon SY, Choi HJ, et al. Association of retinal changes with Alzheimer disease neuroimaging biomarkers in cognitively normal individuals. JAMA Ophthalmol. 2021;139:548-56.

15. Singh RB, Saini C, Shergill S, Agarwal A. Window to the circulatory system: Ocular manifestations of cardiovascular diseases. Eur J Ophthalmol. 2020;30:1207-19.

16. Tan KA, Gupta P, Agarwal A, Chhablani J, Cheng CY, Keane PA, et al. State of science: choroidal thickness and systemic health. Surv Ophthalmol. 2016;61:566-81.

17. Steiner M, Esteban-Ortega MDM, Muñoz-Fernández S. Choroidal and retinal thickness in systemic autoimmune and inflammatory diseases: a review. Surv Ophthalmol. 2019;64:757-69.

18. Akay F, Gundogan FC, Yolcu U, Toyran S, Uzun S. Choroidal thickness in systemic arterial hypertension. Eur J Ophthalmol. 2016;26:152-7. 
19. Dogan Z, Yesildag O, lleri C, Sadic BO, Sunbul M. Evaluation of arterial stiffness and subfoveal choroid thickness in patients with slow coronary flow. Eur Heart J. 2017;38 Suppl 1.

20. Kanar BG, Kanar HS. Relationship between angiographic coronary slow flow phenomenon and subfoveal choroidal thickness: what is the effect of atorvastatin therapy? Eur J Exp Biology. 2018;8:9-14.

21. Wang J, Jiang J, Zhang Y, Qian YW, Zhang JF, Wang ZL. Retinal and choroidal vascular changes in coronary heart disease: an optical coherence tomography angiography study. Biomed Opt Express. 2019;10:1532-44.

22. Amorim Novais E, Badaró E, Allemann N, Ávila Morales MS, Büchele Rodrigues E, de Souza Lima R, et al. Correlation between choroidal thickness and ciliary artery blood flow velocity in normal subjects. Ophthalmic Surg Lasers Imaging Retina. 2015;46:920-4.

23. Frampton JE, Yarker YE, Goa KL. Lenograstim. A review of its pharmacological properties and therapeutic efficacy in neutropenia and related clinical settings. Drugs. 1995;49:767-93.

24. D’Amario D, Leone AM, Borovac JA, Cannata F, Siracusano A, Niccoli G, et al. Granulocyte colonystimulating factor for the treatment of cardiovascular diseases: an update with a critical appraisal. Pharcamol Res. 2018;127:67-76.

25. Orlic D, Kajstura J, Chimenti S, Limana F, Jakoniuk I, Quaini F, et al. Mobilized bone marrow cells repair the infarcted heart, improving function and survival. Proc Natl Acad Sci U S A. 2001;98:103449.

26. Sato D, Otani H, Fujita M, Shimazu T, Yoshioka K, Enoki C, et al. Granulocyte colony-stimulating factor does not enhance recruitment of bone marrow-derived cells in rats with acute myocardial infarction. Exp Clin Cardiol. 2012;17:83-8.

27. Katsaros KM, Speidl WS, Demyanets S, Kastl SP, Krychtiuk KA, Wonnerth A, et al. G-CSF predicts cardiovascular events in patients with stable coronary artery disease. PLOS One. 2015;10:e0142532.

28. Pourtaji A, Jahani V, Moallem SMH, Karimani A, Mohammadpour AH. Application of G-CSF in congestive heart failure treatment. Curr Cardiol Rev. 2019;15:83-90.

29. Singhal A, Subramanian M. Colony stimulating factors (CSFs): complex roles in atherosclerosis. Cytokine. 2019;122:154190.

30. Wilson PW, D'Agostino RB, Levy D, Belanger AM, Silbershatz H, Kannel WB. Prediction of coronary heart disease using risk factor categories. Circulation. 1998;97:1837-47.

31. Conroy RM, Pyorala K, Fitzgerald AP, Sans S, Menotti A, De Backer G, et al. Estimation of ten-tear risk of fatal cardiovascular disease in Europe: the SCORE project. Eur Heart J. 2003;24:987-1003.

32. Hippisley-Cox J, Coupland C, Robson J, Brindle P. Derivation, validation, and evaluation of a new QRISK model to estimate lifetime risk of cardiovascular disease: cohort study using QResearch database. BMJ. 2010;341:c6624.

33. Fernández-Labandera C, Calvo-Bonacho E, Valdivieso P, Quevedo-Aguado L, Martínez-Munoz P, Catalina-Romero $\mathrm{C}$, et al. Prediction of fatal and non-fatal cardiovascular events in Young and middle-aged healthy workers: The IberScore model. Eur J Prev Cardiol. 2021;28:177-86. 
34. Damen JAAG, Hooft L, Schuit E, Debray TP, Collins GS, Tzoulaki I, et al. Prediction models for cardiovascular disease risk in the general population: systematic review. BMJ. 2016;353:i2416.

35. Dadu RT, Nambi V, Ballantyne CM. Developing and assessing cardiovascular biomarkers. Transl Res. 2012;159:265-76.

36. Ciesielski TH, Aldrich MC, Marsit CJ, Hiatt RA, Williams SM. Transdisciplinary approaches enhance the production of translational knowledge. Transl Res. 2017;182:123-34.

\section{Tables}

Table 1: Characteristics of participants according to the presence of coronary lesions.

\begin{tabular}{|lllll|}
\hline & All & $\begin{array}{l}\text { Without } \\
\text { coronary } \\
\text { lesions }\end{array}$ & $\begin{array}{l}\text { With } \\
\text { coronary } \\
\text { lesions }\end{array}$ & $\begin{array}{l}\text { p } \\
\text { (Chi-squared } \\
\text { test) }\end{array}$ \\
\hline NUMBER OF EYES & 192 & 54 & 138 & \\
\hline FEMALE SEX (\%) & $\begin{array}{l}54 / 192 \\
(28)\end{array}$ & $32 / 54(59)$ & $22 / 138(16)$ & $<.001$ \\
\hline AGE IN YEARS (mean \pm SD) & $58.69 \pm 9.49$ & $57.33 \pm 10.67$ & $59.22 \pm 8.93$ & $.22^{\dagger}$ \\
\hline DIABETES (\%) & $\begin{array}{l}68 / 192 \\
(35)\end{array}$ & $18 / 54(33)$ & $50 / 138(36)$ & .71 \\
\hline HIGH BLOOD PRESSURE (\%) & $\begin{array}{l}84 / 192 \\
(44)\end{array}$ & $20 / 54(37)$ & $64 / 138(46)$ & .24 \\
\hline $\begin{array}{l}70 / 192 \\
\text { HYPERCHOLESTEROLEMIA }\end{array}$ & $\begin{array}{l}74 / 54(44) \\
(36)\end{array}$ & $46 / 138(33)$ & .15 \\
\hline SMOKING HABIT (\%) & $\begin{array}{l}78 / 192 \\
(41)\end{array}$ & $12 / 54(22)$ & $66 / 138(48)$ & .001 \\
\hline OBESITY (\%) & $\begin{array}{l}78 / 192 \\
(41)\end{array}$ & $24 / 54(44)$ & $54 / 138(39)$ & .50 \\
\hline
\end{tabular}

SD: Standard deviation.

† Student's t-test.

Table 2: Comparative study for the measured clinical and laboratory ophthalmological variables. 


\begin{tabular}{|c|c|c|c|c|c|}
\hline Variable & Without coronary lesions & $\mathbf{n}$ & $\begin{array}{l}\text { With } \\
\text { coronary lesions }\end{array}$ & $\mathbf{n}$ & $\begin{array}{l}\text { p } \\
\text { (Student's t-test) }\end{array}$ \\
\hline BCVA & $0.95 \pm 0.13$ & 54 & $0.97 \pm 0.07$ & 134 & .36 \\
\hline IOP & $15.13 \pm 3.93$ & 54 & $14.88 \pm 3.11$ & 136 & .64 \\
\hline ST & $16.24 \pm 10.28$ & 54 & $15.20 \pm 9.87$ & 138 & .52 \\
\hline TCCT & $546.28 \pm 32.61$ & 50 & $553.16 \pm 37.76$ & 126 & .26 \\
\hline РCCT & $543.08 \pm 32.95$ & 52 & $549.79 \pm 36.54$ & 130 & .25 \\
\hline $\mathrm{AL}$ & $23.35 \pm 1.48$ & 54 & $23.77 \pm 1.16$ & 120 & .04 \\
\hline CMT & $261.24 \pm 22.77$ & 54 & $267.33 \pm 26.10$ & 132 & .14 \\
\hline MCV & $10.14 \pm 0.39$ & 54 & $10.14 \pm 0.56$ & 132 & .97 \\
\hline MMT & $281.54 \pm 10.83$ & 54 & $281.44 \pm 15.46$ & 132 & .97 \\
\hline RNFLT & $89.54 \pm 9.72$ & 54 & $89.31 \pm 12.39$ & 131 & .90 \\
\hline GCLT & $81.20 \pm 8.44$ & 54 & $79.73 \pm 10.84$ & 133 & .37 \\
\hline GCLMT & $76.63 \pm 12.19$ & 54 & $74.14 \pm 17.36$ & 133 & .34 \\
\hline СТ & $260.74 \pm 44.00$ & 54 & $308.29 \pm 69.92$ & 131 & $<.001$ \\
\hline ILM & $20.68 \pm 4.29$ & 41 & $20.70 \pm 5.40$ & 52 & .99 \\
\hline RNFL-GCL & $21.27 \pm 4.32$ & 41 & $21.45 \pm 5.69$ & 52 & .87 \\
\hline GCL-IPL & $26.08 \pm 4.71$ & 41 & $25.86 \pm 5.73$ & 52 & .85 \\
\hline IPL-INL & $32.64 \pm 5.73$ & 41 & $30.98 \pm 5.52$ & 52 & .16 \\
\hline IL-1b & $0.38 \pm 0.19$ & 39 & $0.45 \pm 0.81$ & 92 & .60 \\
\hline IL-1RA & $4723.71 \pm 2361.33$ & 34 & $4539.61 \pm 2158.62$ & 95 & .68 \\
\hline $\mathrm{IL}-4$ & $0.37 \pm 0.21$ & 44 & $0.35 \pm 0.16$ & 102 & .45 \\
\hline IL-5 & $4.60 \pm 2.75$ & 12 & $3.66 \pm 1.50$ & 30 & .16 \\
\hline IL-6 & $2.54 \pm 3.34$ & 43 & $2.29 \pm 4.00$ & 102 & .73 \\
\hline IL-7 & $4.85 \pm 2.03$ & 45 & $5.01 \pm 2.26$ & 106 & .69 \\
\hline IL-8 & $112.42 \pm 141.78$ & 45 & $66.68 \pm 87.25$ & 107 & .02 \\
\hline IL-9 & $4.57 \pm 3.38$ & 29 & $4.84 \pm 3.06$ & 59 & .70 \\
\hline IL-13 & $0.43 \pm 0.25$ & 25 & $0.40 \pm 0.21$ & 75 & .60 \\
\hline IL-17 & $2.37 \pm 0.97$ & 2 & $2.10 \pm 1.80$ & 14 & .85 \\
\hline
\end{tabular}




\begin{tabular}{|llllll|}
\hline EO & $0.62 \pm 0.23$ & 45 & $0.58 \pm 0.22$ & 107 & .24 \\
\hline G-CSF & $\mathbf{4 4 . 3 8} \pm 53.10$ & 25 & $20.98 \pm 29.01$ & 59 & .01 \\
\hline IFN-g & $65.86 \pm 23.69$ & 28 & $0.62 \pm 0.28$ & 75 & .57 \\
\hline IP-10 & $2062.35 \pm 1174.51$ & 45 & $58.62 \pm 24.41$ & 107 & .09 \\
\hline MCP-1 & $21.67 \pm 20.85$ & 42 & $2338.23 \pm 1109.79$ & 100 & .19 \\
\hline MIP-1a & $0.74 \pm 1.51$ & 45 & $13.72 \pm 16.48$ & 107 & .01 \\
\hline PDGF-BB & $19.49 \pm 11.51$ & 45 & $0.39 \pm 0.61$ & 106 & .05 \\
\hline MIP-1b & $4.06 \pm 3.92$ & 18 & $21.16 \pm 11.49$ & 35 & .62 \\
\hline RANTES & $22.36 \pm 15.69$ & 42 & $4.25 \pm 6.68$ & 97 & .87 \\
\hline TNF-a & $13.26 \pm 11.69$ & 45 & $33.85 \pm 27.22$ & 107 & .009 \\
\hline
\end{tabular}

Data are shown as mean \pm standard deviation.

n: Number of eyes. BCVA: Best corrected visual acuity. IOP: Intraocular pressure. ST: Schirmer's test. TCCT: Topography central corneal thickness. PCCT: Pachymetry central corneal thickness. AL: Axial length. CMT: Central macular thickness. MCV: Macular cube volume. MMT: Mean macular thickness. RNFLT: Retinal nerve fibre layer thickness. GCLT: Ganglion cell layer thickness. GCLMT: Ganglion cell layer minimun thickness. CT: Choroidal thickness. ILM: Central vascular density under the inner limiting membrane. RNFL-GCL: Central vascular density between retinal nerve fibre layer and ganglion cell layer. GCL-IPL: Central vascular density between ganglion cell layer and inner plexiform layer. IPL-INL: Central vascular density between inner plexiform layer and inner nuclear layer. IL: Interleukin. EO: Eotaxin. G-CSF: Granulocyte colony-stimulating factor. GM-CSF: Granulocyte-macrophage colony-stimulating factor. IFN: Interferon. IP: Chemokine. MCP: Monocyte chemoattractant protein. MIP: Macrophage inflammatory protein. PDGF: Platelet-derived growth factor. RANTES: Chemokine ligand 5. TNF: Tumour necrosis factor.

Table 3: Logistic regression and comparative study between area under curve (AUC) of the prediction models with the classic risk factors (CRF) including, or not, the selected ophthalmological variables. 


\begin{tabular}{|c|c|c|c|}
\hline Variable & $\mathrm{OR}^{\dagger}(\mathrm{Cl} 95 \%)$ & AUC§ (Cl 95\%) & AUC' (Cl 95\%) \\
\hline AL & $1.13(0.81-1.58)$ & $0.83(0.76-0.90)$ & $0.83(0.76-0.90)$ \\
\hline CT & $1.02^{\ddagger}(1.01-1.03)$ & $0.89^{a}(0.84-0.94)$ & $0.83(0.77-0.90)$ \\
\hline IL-8 & $0.99 \ddagger(0.99-1.00)$ & $0.86(0.80-0.92)$ & $0.84(0.78-0.91)$ \\
\hline G-CSF & $0.97^{\ddagger}(0.95-0.99)$ & $0.91^{a}(0.84-0.97)$ & $0.82(0.72-0.92)$ \\
\hline MCP-1 & $0.96^{\ddagger}(0.94-0.99)$ & $0.87(0.80-0.93)$ & $0.84(0.78-0.91)$ \\
\hline MIP-1b & $0.99(0.91-1.08)$ & $0.87(0.80-0.93)$ & $0.85(0.79-0.92)$ \\
\hline RANTES & $1.02^{\ddagger}(1.00-1.05)$ & $0.87(0.81-0.93)$ & $0.84(0.78-0.91)$ \\
\hline
\end{tabular}

OR: Odds ratio. Cl: Confidence interval. AL: Axial length. CT: Choroidal thickness. IL: Interleukin. G-CSF: Granulocyte colony-stimulating factor. MCP: Monocyte chemoattractant protein. MIP: Macrophage inflammatory protein. RANTES: Chemokine ligand 5.

${ }^{\dagger}$ Adjusted by variable and CRF: sex, age, diabetes, high blood pressure, hypercholesterolemia, smoking habit, and obesity.

${ }^{\ddagger} p<.05$ (likelihood ratio test).

§ AUC from model adjusted by variable and CRF.

'AUC from model adjusted only by CRF.

Statistically significantly $(p<.05)$ higher than AUC from model adjusted only by CRF.

Table 4: Area under curve (AUC) for the prediction models with the classic risk factors (CRF) including, or not, the selected ophthalmological variables.

\begin{tabular}{|llll|}
\hline Variables & $\mathrm{AUC}^{\dagger}(\mathrm{Cl}$ 95\%) & $\mathrm{AUC}^{\ddagger}(\mathrm{Cl} 95 \%)$ & $\mathrm{p}$ (Chi-square test) \\
\hline $\mathrm{CT}+\mathrm{G}-\mathrm{CSF}$ & $0.964(0.928-0.999)$ & $0.828(0.729-0.927)$ & .003 \\
\hline
\end{tabular}

Cl: Confidence interval. CT: Choroidal thickness. G-CSF: Granulocyte colony-stimulating factor.

${ }^{\dagger}$ AUC from model adjusted by CT, G-CSF and CRF: sex, age, diabetes, high blood pressure, hypercholesterolemia, smoking habit, and obesity.

‡ AUC from model adjusted only by CRF.

\section{Figures}




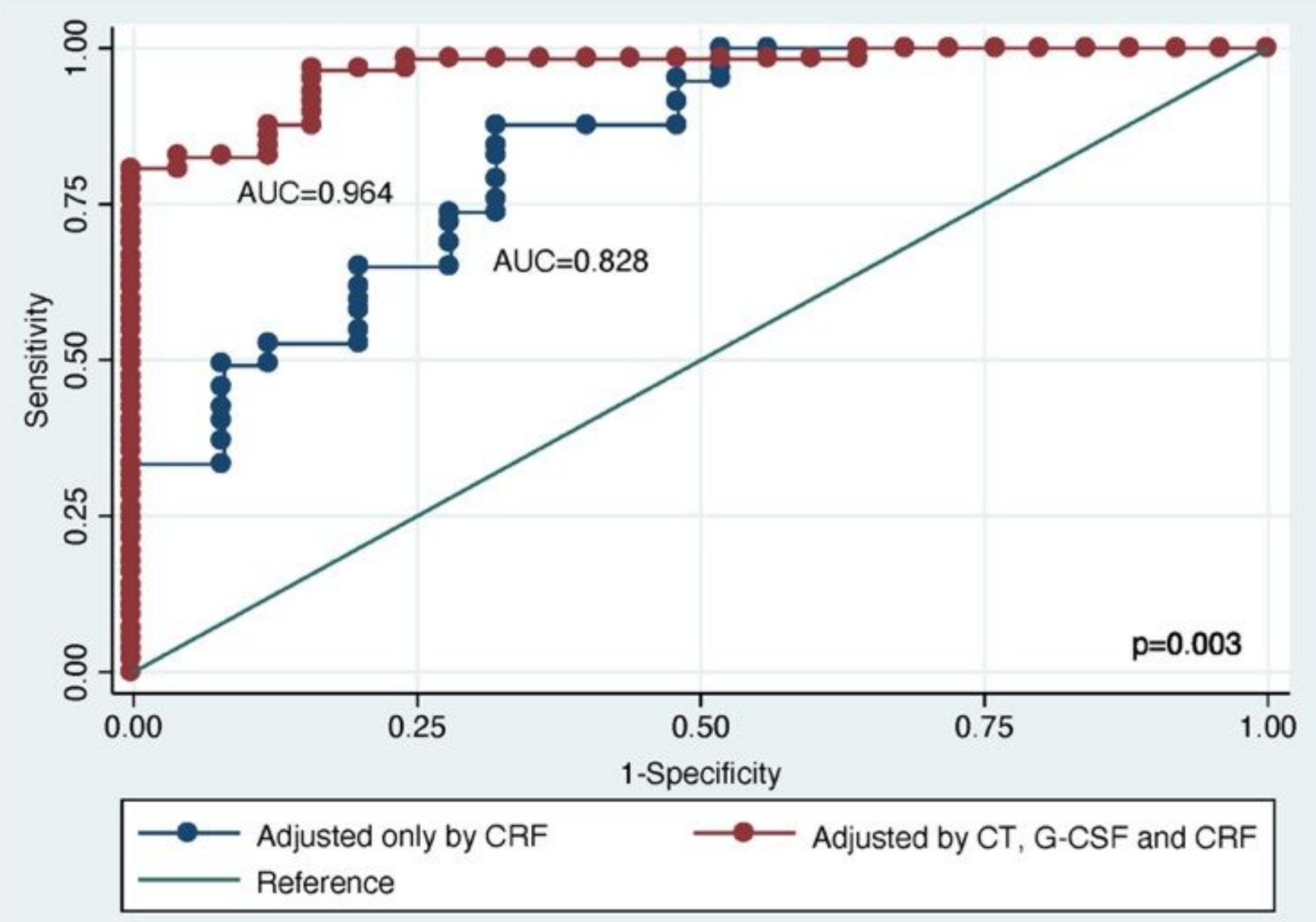

Figure 1

Comparison of receiver operating characteristic (ROC) curves between logistic regression models.

AUC: Area under curve. CRF: Classic risk factors. CT: Choroidal thickness, G-CSF: Granulocyte colonystimulating factor.

p-Value: Chi-square test. 